\title{
Combined Theoretical and Experimental Deep-UV Resonance Raman Studies of Substituted Pyrenes
}

\section{Supplementary Material}

\author{
Johannes Neugebauer, Evert Jan Baerends ${ }^{1}$ \\ Theoretical Chemistry, Vrije Universiteit Amsterdam \\ De Boelelaan 1083, 1081 HV Amsterdam, The Netherlands \\ Evtim V. Efremov, Freek Ariese, Cees Gooijer ${ }^{2}$ \\ Analytical Chemistry \& Applied Spectroscopy, Vrije Universiteit Amsterdam, \\ De Boelelaan 1083, 1081 HV Amsterdam, The Netherlands
}

Date: December 22, 2004

Status: revised version, submitted to J. Phys. Chem. A

\footnotetext{
${ }^{1}$ email: $\{$ jneugeb,baerends\}@chem.vu.nl

${ }^{2}$ email: $\{$ e.efremov,ariese,gooijer $\} @$ few.vu.nl
} 
Table S1: Calculated vibrational frequencies (BP86/TZP) in units of $\mathrm{cm}^{-1}$ for 1-fluoropyrene.

\begin{tabular}{|c|c|c|c|c|c|c|c|c|}
\hline no. & irrep & $\tilde{\nu}$ & no. & irrep & $\tilde{\nu}$ & no. & irrep & $\tilde{\nu}$ \\
\hline 1 & $a^{\prime \prime}$ & 86 & 25 & $a^{\prime}$ & 760 & 49 & $a^{\prime}$ & 1313 \\
\hline 2 & $a^{\prime \prime}$ & 117 & 26 & $a^{\prime \prime}$ & 775 & 50 & $a^{\prime}$ & 1338 \\
\hline 3 & $a^{\prime \prime}$ & 197 & 27 & $a^{\prime \prime}$ & 805 & 51 & $a^{\prime}$ & 1376 \\
\hline 4 & $a^{\prime \prime}$ & 220 & 28 & $a^{\prime}$ & 813 & 52 & $a^{\prime}$ & 1389 \\
\hline 5 & $a^{\prime}$ & 251 & 29 & $a^{\prime \prime}$ & 816 & 53 & $a^{\prime}$ & 1397 \\
\hline 6 & $a^{\prime \prime}$ & 256 & 30 & $a^{\prime \prime}$ & 824 & 54 & $a^{\prime}$ & 1406 \\
\hline 7 & $a^{\prime \prime}$ & 314 & 31 & $a^{\prime \prime}$ & 881 & 55 & $a^{\prime}$ & 1420 \\
\hline 8 & $a^{\prime}$ & 378 & 32 & $a^{\prime}$ & 881 & 56 & $a^{\prime}$ & 1448 \\
\hline 9 & $a^{\prime}$ & 413 & 33 & $a^{\prime \prime}$ & 912 & 57 & $a^{\prime}$ & 1475 \\
\hline 10 & $a^{\prime \prime}$ & 422 & 34 & $a^{\prime \prime}$ & 945 & 58 & $a^{\prime}$ & 1494 \\
\hline 11 & $a^{\prime}$ & 439 & 35 & $a^{\prime \prime}$ & 951 & 59 & $a^{\prime}$ & 1547 \\
\hline 12 & $a^{\prime}$ & 477 & 36 & $a^{\prime \prime}$ & 956 & 60 & $a^{\prime}$ & 1579 \\
\hline 13 & $a^{\prime \prime}$ & 490 & 37 & $a^{\prime}$ & 965 & 61 & $a^{\prime}$ & 1589 \\
\hline 14 & $a^{\prime \prime}$ & 504 & 38 & $a^{\prime}$ & 1052 & 62 & $a^{\prime}$ & 1595 \\
\hline 15 & $a^{\prime}$ & 515 & 39 & $a^{\prime}$ & 1090 & 63 & $a^{\prime}$ & 1618 \\
\hline 16 & $a^{\prime \prime}$ & 538 & 40 & $a^{\prime}$ & 1099 & 64 & $a^{\prime}$ & 3085 \\
\hline 17 & $a^{\prime}$ & 542 & 41 & $a^{\prime}$ & 1134 & 65 & $a^{\prime}$ & 3090 \\
\hline 18 & $a^{\prime \prime}$ & 597 & 42 & $a^{\prime}$ & 1146 & 66 & $a^{\prime}$ & 3094 \\
\hline 19 & $a^{\prime}$ & 598 & 43 & $a^{\prime}$ & 1157 & 67 & $a^{\prime}$ & 3096 \\
\hline 20 & $a^{\prime \prime}$ & 671 & 44 & $a^{\prime}$ & 1171 & 68 & $a^{\prime}$ & 3101 \\
\hline 21 & $a^{\prime}$ & 686 & 45 & $a^{\prime}$ & 1205 & 69 & $a^{\prime}$ & 3106 \\
\hline 22 & $a^{\prime}$ & 689 & 46 & $a^{\prime}$ & 1223 & 70 & $a^{\prime}$ & 3115 \\
\hline 23 & $a^{\prime \prime}$ & 701 & 47 & $a^{\prime}$ & 1237 & 71 & $a^{\prime}$ & 3132 \\
\hline 24 & $a^{\prime \prime}$ & 742 & 48 & $a^{\prime}$ & 1239 & 72 & $a^{\prime}$ & 3134 \\
\hline
\end{tabular}


Table S2: Calculated vibrational frequencies (BP86/TZP) in units of $\mathrm{cm}^{-1}$ for 1-chloropyrene.

\begin{tabular}{rrr|rrr|rrr}
\hline \hline no. & irrep & $\tilde{\nu}$ & no. & irrep & $\tilde{\nu}$ & no. & irrep & $\tilde{\nu}$ \\
\hline 1 & $a^{\prime \prime}$ & 71 & 25 & $a^{\prime}$ & 748 & 49 & $a^{\prime}$ & 1307 \\
2 & $a^{\prime \prime}$ & 105 & 26 & $a^{\prime \prime}$ & 775 & 50 & $a^{\prime}$ & 1329 \\
3 & $a^{\prime \prime}$ & 168 & 27 & $a^{\prime \prime}$ & 805 & 51 & $a^{\prime}$ & 1366 \\
4 & $a^{\prime}$ & 193 & 28 & $a^{\prime}$ & 813 & 52 & $a^{\prime}$ & 1381 \\
5 & $a^{\prime \prime}$ & 205 & 29 & $a^{\prime \prime}$ & 821 & 53 & $a^{\prime}$ & 1396 \\
6 & $a^{\prime \prime}$ & 254 & 30 & $a^{\prime}$ & 824 & 54 & $a^{\prime}$ & 1405 \\
7 & $a^{\prime \prime}$ & 292 & 31 & $a^{\prime \prime}$ & 832 & 55 & $a^{\prime}$ & 1417 \\
8 & $a^{\prime}$ & 325 & 32 & $a^{\prime \prime}$ & 882 & 56 & $a^{\prime}$ & 1448 \\
9 & $a^{\prime}$ & 401 & 33 & $a^{\prime \prime}$ & 922 & 57 & $a^{\prime}$ & 1468 \\
10 & $a^{\prime}$ & 409 & 34 & $a^{\prime \prime}$ & 946 & 58 & $a^{\prime}$ & 1487 \\
11 & $a^{\prime \prime}$ & 410 & 35 & $a^{\prime \prime}$ & 952 & 59 & $a^{\prime}$ & 1539 \\
12 & $a^{\prime}$ & 473 & 36 & $a^{\prime \prime}$ & 957 & 60 & $a^{\prime}$ & 1574 \\
13 & $a^{\prime \prime}$ & 488 & 37 & $a^{\prime}$ & 965 & 61 & $a^{\prime}$ & 1580 \\
14 & $a^{\prime \prime}$ & 502 & 38 & $a^{\prime}$ & 1009 & 62 & $a^{\prime}$ & 1594 \\
15 & $a^{\prime}$ & 509 & 39 & $a^{\prime}$ & 1076 & 63 & $a^{\prime}$ & 1616 \\
16 & $a^{\prime}$ & 527 & 40 & $a^{\prime}$ & 1101 & 64 & $a^{\prime}$ & 3089 \\
17 & $a^{\prime \prime}$ & 532 & 41 & $a^{\prime}$ & 1130 & 65 & $a^{\prime}$ & 3092 \\
18 & $a^{\prime}$ & 576 & 42 & $a^{\prime}$ & 1138 & 66 & $a^{\prime}$ & 3096 \\
\hline \hline
\end{tabular}


Table S3: Calculated vibrational frequencies (BP86/TZP) in units of $\mathrm{cm}^{-1}$ for 1-bromopyrene.

\begin{tabular}{|c|c|c|c|c|c|c|c|c|}
\hline no. & irrep & $\tilde{\nu}$ & no. & irrep & $\tilde{\nu}$ & no. & irrep & $\tilde{\nu}$ \\
\hline 1 & $a^{\prime \prime}$ & 71 & 25 & $a^{\prime}$ & 742 & 49 & $a^{\prime}$ & 1304 \\
\hline 2 & $a^{\prime \prime}$ & 108 & 26 & $a^{\prime \prime}$ & 772 & 50 & $a^{\prime}$ & 1329 \\
\hline 3 & $a^{\prime}$ & 144 & 27 & $a^{\prime \prime}$ & 803 & 51 & $a^{\prime}$ & 1365 \\
\hline 4 & $a^{\prime \prime}$ & 177 & 28 & $a^{\prime}$ & 805 & 52 & $a^{\prime}$ & 1377 \\
\hline 5 & $a^{\prime \prime}$ & 217 & 29 & $a^{\prime}$ & 812 & 53 & $a^{\prime}$ & 1395 \\
\hline 6 & $a^{\prime}$ & 238 & 30 & $a^{\prime \prime}$ & 823 & 54 & $a^{\prime}$ & 1404 \\
\hline 7 & $a^{\prime \prime}$ & 261 & 31 & $a^{\prime \prime}$ & 832 & 55 & $a^{\prime}$ & 1415 \\
\hline 8 & $a^{\prime \prime}$ & 285 & 32 & $a^{\prime \prime}$ & 882 & 56 & $a^{\prime}$ & 1447 \\
\hline 9 & $a^{\prime}$ & 387 & 33 & $a^{\prime \prime}$ & 921 & 57 & $a^{\prime}$ & 1463 \\
\hline 10 & $a^{\prime}$ & 405 & 34 & $a^{\prime \prime}$ & 947 & 58 & $a^{\prime}$ & 1485 \\
\hline 11 & $a^{\prime \prime}$ & 409 & 35 & $a^{\prime \prime}$ & 952 & 59 & $a^{\prime}$ & 1539 \\
\hline 12 & $a^{\prime}$ & 467 & 36 & $a^{\prime \prime}$ & 957 & 60 & $a^{\prime}$ & 1571 \\
\hline 13 & $a^{\prime \prime}$ & 484 & 37 & $a^{\prime}$ & 963 & 61 & $a^{\prime}$ & 1579 \\
\hline 14 & $a^{\prime \prime}$ & 504 & 38 & $a^{\prime}$ & 992 & 62 & $a^{\prime}$ & 1594 \\
\hline 15 & $a^{\prime}$ & 505 & 39 & $a^{\prime}$ & 1072 & 63 & $a^{\prime}$ & 1615 \\
\hline 16 & $a^{\prime}$ & 519 & 40 & $a^{\prime}$ & 1101 & 64 & $a^{\prime}$ & 3090 \\
\hline 17 & $a^{\prime \prime}$ & 532 & 41 & $a^{\prime}$ & 1121 & 65 & $a^{\prime}$ & 3090 \\
\hline 18 & $a^{\prime}$ & 563 & 42 & $a^{\prime}$ & 1140 & 66 & $a^{\prime}$ & 3095 \\
\hline 19 & $a^{\prime \prime}$ & 580 & 43 & $a^{\prime}$ & 1152 & 67 & $a^{\prime}$ & 3099 \\
\hline 20 & $a^{\prime}$ & 606 & 44 & $a^{\prime}$ & 1170 & 68 & $a^{\prime}$ & 3100 \\
\hline 21 & $a^{\prime \prime}$ & 672 & 45 & $a^{\prime}$ & 1188 & 69 & $a^{\prime}$ & 3109 \\
\hline 22 & $a^{\prime}$ & 688 & 46 & $a^{\prime}$ & 1213 & 70 & $a^{\prime}$ & 3115 \\
\hline 23 & $a^{\prime \prime}$ & 699 & 47 & $a^{\prime}$ & 1233 & 71 & $a^{\prime}$ & 3130 \\
\hline 24 & $a^{\prime \prime}$ & 740 & 48 & $a^{\prime}$ & 1237 & 72 & $a^{\prime}$ & 3134 \\
\hline
\end{tabular}


Table S4: Experimental $\left(E_{\exp }\right)$ and calculated (SAOP/TZP; $\left.E_{\exp }\right)$ vertical excitation energies for the lowest dipole-allowed transitions of pyrene. Also shown are calculated oscillator strengths.

\begin{tabular}{rrrrrrr}
\hline \hline no. & state & $E_{\text {exp }} / \mathrm{eV}$ & $E_{\text {calc }} / \mathrm{eV}$ & $E_{\text {exp }} / \mathrm{nm}$ & $E_{\text {calc }} / \mathrm{nm}$ & $\mathrm{f}$ \\
\hline 1 & $1 B_{2 u}$ & 3.72 & 3.38 & 333 & 367 & 0.20831 \\
2 & $1 B_{3 u}$ & 3.33 & 3.46 & 372 & 358 & 0.00004 \\
3 & $2 B_{3 u}$ & 4.57 & 4.17 & 272 & 297 & 0.17832 \\
4 & $2 B_{2 u}$ & 5.18 & 5.07 & 239 & 245 & 0.57335 \\
5 & $1 B_{1 u}$ & & 5.25 & & 236 & 0.00001 \\
6 & $3 B_{3 u}$ & & 5.70 & & 218 & 0.00537 \\
7 & $3 B_{2 u}$ & & 5.87 & & 211 & 0.63229 \\
\hline \hline
\end{tabular}


Table S5: Experimental ( $\left.E_{\exp }\right)$ and calculated (SAOP/TZP; $\left.E_{\exp }\right)$ vertical excitation energies for the lowest dipole-allowed transitions of 1-fluoropyrene. Also shown are calculated oscillator strengths.

\begin{tabular}{|c|c|c|c|c|c|c|}
\hline no. & state & $E_{\text {exp }} / \mathrm{eV}$ & $E_{\text {calc }} / \mathrm{eV}$ & $E_{\exp } / \mathrm{nm}$ & $E_{\text {calc }} / \mathrm{nm}$ & $\mathrm{f}$ \\
\hline 1 & $1 A^{\prime}$ & 3.72 & 3.34 & 333 & 371 & 0.19514 \\
\hline 2 & $2 A^{\prime}$ & 3.31 & 3.45 & 375 & 359 & 0.00967 \\
\hline 3 & $3 A^{\prime}$ & & 3.84 & & 323 & 0.00330 \\
\hline 4 & $4 A^{\prime}$ & 4.56 & 4.14 & 272 & 299 & 0.16472 \\
\hline 5 & $5 A^{\prime}$ & & 4.20 & & 295 & 0.00213 \\
\hline 6 & $6 A^{\prime}$ & & 4.42 & & 281 & 0.01050 \\
\hline 7 & $7 A^{\prime}$ & & 4.71 & & 263 & 0.00356 \\
\hline 8 & $8 A^{\prime}$ & 5.19 & 5.04 & 239 & 246 & 0.50127 \\
\hline 9 & $9 A^{\prime}$ & & 5.07 & & 245 & 0.01868 \\
\hline 10 & $10 A^{\prime}$ & & 5.15 & & 241 & 0.01734 \\
\hline 11 & $11 A^{\prime}$ & & 5.22 & & 238 & 0.03883 \\
\hline 12 & $1 A^{\prime \prime}$ & & 5.36 & & 231 & 0.00000 \\
\hline 13 & $12 A^{\prime}$ & & 5.42 & & 229 & 0.00041 \\
\hline 14 & $2 A^{\prime \prime}$ & & 5.48 & & 226 & 0.00002 \\
\hline 15 & $13 A^{\prime}$ & & 5.52 & & 225 & 0.00076 \\
\hline 16 & $3 A^{\prime \prime}$ & & 5.56 & & 223 & 0.00004 \\
\hline 17 & $4 A^{\prime \prime}$ & & 5.66 & & 219 & 0.00002 \\
\hline 18 & $14 A^{\prime}$ & & 5.67 & & 219 & 0.03611 \\
\hline 19 & $15 A^{\prime}$ & & 5.89 & & 210 & 0.52402 \\
\hline 20 & $16 A^{\prime}$ & & 5.90 & & 210 & 0.03185 \\
\hline
\end{tabular}


Table S6: Experimental $\left(E_{\exp }\right)$ and calculated (SAOP/TZP; $\left.E_{\exp }\right)$ vertical excitation energies for the lowest dipole-allowed transitions of 1-chloropyrene. Also shown are calculated oscillator strengths.

\begin{tabular}{|c|c|c|c|c|c|c|}
\hline no. & state & $E_{\exp } / \mathrm{eV}$ & $E_{\text {calc }} / \mathrm{eV}$ & $E_{\exp } / \mathrm{nm}$ & $E_{\text {calc }} / \mathrm{nm}$ & f \\
\hline 1 & $1 A^{\prime}$ & 3.63 & 3.27 & 342 & 379 & 0.23794 \\
\hline 2 & $2 A^{\prime}$ & 3.28 & 3.41 & 377 & 364 & 0.00642 \\
\hline 3 & $3 A^{\prime}$ & & 3.82 & & 325 & 0.00257 \\
\hline 4 & $4 A^{\prime}$ & 4.51 & 4.09 & 275 & 303 & 0.17539 \\
\hline 5 & $5 A^{\prime}$ & & 4.12 & & 301 & 0.00005 \\
\hline 6 & $6 A^{\prime}$ & & 4.30 & & 288 & 0.01005 \\
\hline 7 & $7 A^{\prime}$ & & 4.69 & & 264 & 0.00215 \\
\hline 8 & $8 A^{\prime}$ & & 4.83 & & 257 & 0.07532 \\
\hline 9 & $1 A^{\prime \prime}$ & & 4.88 & & 254 & 0.00000 \\
\hline 10 & $2 A^{\prime \prime}$ & & 4.93 & & 251 & 0.00001 \\
\hline 11 & $9 A^{\prime}$ & & 4.97 & & 249 & 0.25114 \\
\hline 12 & $10 A^{\prime}$ & & 5.10 & & 243 & 0.09696 \\
\hline 13 & $11 A^{\prime}$ & 5.14 & 5.11 & 241 & 243 & 0.16060 \\
\hline 14 & $3 A^{\prime \prime}$ & & 5.32 & & 233 & 0.00000 \\
\hline 15 & $12 A^{\prime}$ & & 5.33 & & 233 & 0.00264 \\
\hline 16 & $13 A^{\prime}$ & & 5.35 & & 232 & 0.02958 \\
\hline 17 & $4 A^{\prime \prime}$ & & 5.51 & & 225 & 0.00000 \\
\hline 18 & $14 A^{\prime}$ & & 5.54 & & 224 & 0.03706 \\
\hline 19 & $15 A^{\prime}$ & & 5.71 & & 217 & 0.12805 \\
\hline 20 & $16 A^{\prime}$ & & 5.74 & & 216 & 0.00941 \\
\hline
\end{tabular}


Table S7: Experimental ( $\left.E_{\exp }\right)$ and calculated (SAOP/TZP; $\left.E_{\exp }\right)$ vertical excitation energies for the lowest dipole-allowed transitions of 1-bromopyrene. Also shown are calculated oscillator strengths.

\begin{tabular}{|c|c|c|c|c|c|c|}
\hline no. & state & $E_{\exp } / \mathrm{eV}$ & $E_{\text {calc }} / \mathrm{eV}$ & $E_{\exp } / \mathrm{nm}$ & $E_{\text {calc }} / \mathrm{nm}$ & f \\
\hline 1 & $1 A^{\prime}$ & 3.61 & 3.24 & 343 & 383 & 0.24416 \\
\hline 2 & $2 A^{\prime}$ & 3.29 & 3.40 & 377 & 365 & 0.00928 \\
\hline 3 & $3 A^{\prime}$ & & 3.81 & & 325 & 0.00304 \\
\hline 4 & $4 A^{\prime}$ & 4.49 & 4.04 & 276 & 307 & 0.11895 \\
\hline 5 & $1 A^{\prime \prime}$ & & 4.05 & & 306 & 0.00001 \\
\hline 6 & $5 A^{\prime}$ & & 4.10 & & 302 & 0.04758 \\
\hline 7 & $6 A^{\prime}$ & & 4.25 & & 292 & 0.01913 \\
\hline 8 & $2 A^{\prime \prime}$ & & 4.30 & & 288 & 0.00002 \\
\hline 9 & $7 A^{\prime}$ & & 4.62 & & 268 & 0.03147 \\
\hline 10 & $8 A^{\prime}$ & & 4.68 & & 265 & 0.00248 \\
\hline 11 & $9 A^{\prime}$ & & 4.84 & & 256 & 0.09117 \\
\hline 12 & $3 A^{\prime \prime}$ & & 4.84 & & 256 & 0.00013 \\
\hline 13 & $4 A^{\prime \prime}$ & & 5.06 & & 245 & 0.00008 \\
\hline 14 & $10 A^{\prime}$ & 5.12 & 5.07 & 242 & 245 & 0.28452 \\
\hline 15 & $11 A^{\prime}$ & & 5.09 & & 244 & 0.17662 \\
\hline 16 & $12 A^{\prime}$ & & 5.22 & & 238 & 0.02502 \\
\hline 17 & $5 A^{\prime \prime}$ & & 5.26 & & 236 & 0.00000 \\
\hline 18 & $13 A^{\prime}$ & & 5.32 & & 233 & 0.01509 \\
\hline 19 & $14 A^{\prime}$ & & 5.42 & & 229 & 0.02729 \\
\hline 20 & $15 A^{\prime}$ & & 5.54 & & 224 & 0.00120 \\
\hline
\end{tabular}

